Research Article

\title{
A Modified Proximal Point Algorithm and Some Convergence Results
}

\author{
Shengquan Weng $\mathbb{D}^{1},{ }^{1}$ Dingping $\mathrm{Wu}^{2}$, and Zengfu Chao ${ }^{1}$ \\ ${ }^{1}$ Faculty of Physics and Mathematics, Yibin University, Yibin 644000, China \\ ${ }^{2}$ Department of Applied Mathematics, Chengdu University of Information Technology, Chengdu 610225, China
}

Correspondence should be addressed to Shengquan Weng; ssldstg@qq.com

Received 20 September 2021; Revised 17 October 2021; Accepted 21 October 2021; Published 15 November 2021

Academic Editor: Jen-Chih Yao

Copyright (c) 2021 Shengquan Weng et al. This is an open access article distributed under the Creative Commons Attribution License, which permits unrestricted use, distribution, and reproduction in any medium, provided the original work is properly cited.

In this paper, the convergence to minimizers of a convex function of a modified proximal point algorithm involving a singlevalued nonexpansive mapping and a multivalued nonexpansive mapping in CAT(0) spaces is studied and a numerical example is given to support our main results.

\section{Introduction}

In daily life, no matter what we do, there are always many options available and many possible outcomes. When we do these things, we always consciously or unconsciously choose an optimal solution in order to achieve the optimal result. The discipline of seeking the best solution to achieve the best result is optimization. The way to find the optimal solution is the optimization method.

Given a real number $\kappa$ (curvature), let $M_{\kappa}^{2}$ denote the following space: if $\kappa<0$, then $M_{\kappa}^{2}$ is a real hyperbolic space $H^{2}$ with the distance function scaled by a factor of $(1 / \sqrt{-\kappa})$; if $\kappa=0$, then $M_{\kappa}^{2}$ is the Euclidean plane; if $\kappa>0$, then $M_{\kappa}^{2}$ is the 2 -sphere $S^{2}$ with the metric scaled by a factor $(1 / \sqrt{\kappa})$. Let $D_{\kappa}$ denote the diameter of $M_{\kappa}^{2}$. Let $\Delta$ be a geodesic triangle in $X$ with a perimeter less than $2 D_{\kappa}$. Let $\bar{\Delta} \in M_{\kappa}^{2}$ be a comparison triangle for $\bar{\Delta}$. Then, $\Delta$ is said to satisfy the CAT $(\kappa)$ inequality if for $x, y \in \Delta$ and all comparison points $\bar{x}, \bar{y} \in \bar{\Delta}$,

$$
d(x, y) \leq d(\bar{x}, \bar{y})
$$

If $\kappa \leq 0$, then $X$ is called a $\operatorname{CAT}(\kappa)$ space if $X$ is a geodesic space all of whose geodesic triangles satisfy the $\mathrm{CAT}(\kappa)$ inequality. If $\kappa>0$, then $X$ is called a CAT $(\kappa)$ space if $X$ is $D_{\kappa}$-geodesic and all geodesic triangles in $X$ of perimeter less than $2 D_{\kappa}$ satisfy the CAT $(\kappa)$ inequality. Thus, it can be seen that a CAT(0) space is a special case of $\mathrm{CAT}(\kappa)$ spaces when the curvature $\kappa=0$. Furthermore, it is possible that the metric on $\mathrm{CAT}(\kappa)$ spaces $(\kappa>0)$ may take infinite values.

A metric space is said to be a geodesic metric space if every two points of $X$ are joined by a geodesic in this metric space; a geodesic metric space $(X, d)$ is said to be a CAT $(0)$ space if each geodesic triangle of geodesic metric space $(X, d)$ is at least as "thin" as its comparison triangle in $R^{2}$. In addition, a CAT(0) space is said to be a Hadamard space if it is complete; see for more details in [1-8]. Let $X$ be a geodesic metric space and $D$ be a nonempty subset of $X$. One of the major problems for optimization is to find a point $x \in X$ such that

$$
f(x)=\min _{y \in X} f(y)
$$

where $f$ is a proper convex lower semicontinuous function and the set of all minimizers of $f$ on $X$ is denoted by $\operatorname{argmin}_{y \in X} f(y)$. There are many ways to study this problem.

For any $\lambda>0, \forall x \in X$, the Moreau-Yosida resolvent of $f$ is defined in $\operatorname{CAT}(0)$ spaces $X$ as

$$
J_{\lambda}(x)=\underset{y \in X}{\arg \min }\left[f(y)+\frac{1}{2 \lambda} d^{2}(y, x)\right] .
$$


In fact, here $f$ is a proper convex and lower semicontinuous function. The concept of Moreau - Yosida resolvent of $f$ appeared in [9]. Furthermore, the fact that the set $F\left(J_{\lambda}\right)$ of fixed points of the resolvent associated with $f$ coincides with the set $\operatorname{argmin}_{y \in X} f(y)$ of minimizers of $f$ is shown in $[10,11]$. Also, for any $\lambda>0$, the resolvent $J_{\lambda}$ of $f$ is nonexpansive [12].

In 1970, Martinet [13] published an article and a new algorithm was proposed to solve this optimization problem; the new algorithm is called as the proximal point algorithm. In 1976, in a Hilbert space, Rockafellar [14] studied the convergence to a solution of the convex minimization problem by the proximal point algorithm and proved and obtained a main conclusion that the sequence $\left\{x_{n}\right\}$ converges weakly to a minimizer of a convex function $f$ such that $\sum_{n=1}^{\infty} \lambda_{n}=\infty$. In 2013, the proximal point algorithm was introduced by Bacák [15] into $\operatorname{CAT}(0)$ spaces $(X, d)$ as follows: $x_{1} \in X$, for each $n \in N$,

$$
x_{n+1}=\underset{y \in X}{\arg \min }\left[f(y)+\frac{1}{2 \lambda_{n}} d^{2}\left(y, x_{n}\right)\right] .
$$

Here, $\lambda_{n}>0, \forall n \in N$, and this shows that, if $f$ has a minimizer, $\sum_{n=1}^{\infty} \lambda_{n}=\infty$, then the sequence $\left\{x_{n}\right\} \Delta$-converges to its minimizer. In fact, the proximal point algorithm has been combined with many iterative methods, and a new construction algorithm is further proposed to find approximating fixed points of nonlinear mappings and a proper convex lower semicontinuous function $f$. In 1953, a known iteration method was proposed by Mann, and it was named as Mann iteration [16]; the Mann iteration process is defined as follows: $x_{1} \in D$ and

$$
x_{n+1}=\alpha_{n} x_{n}+\left(1-\alpha_{n}\right) T x_{n} .
$$

Here, $\left\{\alpha_{n}\right\}, n \in N$, is a real sequence in $(0,1)$. In 1974 , another well-known iteration method was proposed by Ishikawa, and this iteration method was named as Ishikawa iteration [17]; the Ishikawa iteration process concretely is expressed as follows: $x_{1} \in D$ and

$$
\left\{\begin{array}{l}
y_{n}=\left(1-\alpha_{n}\right) T x_{n}+\alpha_{n} x_{n} \\
x_{n+1}=\left(1-\beta_{n}\right) T y_{n}+\beta_{n} x_{n}
\end{array}\right.
$$

for each $n \in N$, where $\left\{\alpha_{n}\right\},\left\{\beta_{n}\right\}$ is a real sequence in $(0,1)$.

In 2017, Suthep Suantai and Withum Phuengtattana [18] proposed a proximal point algorithm for a hybrid pair of nonexpansive single-valued and multivalued mappings in geodesic metric spaces as follows:

$$
\left\{\begin{array}{l}
z_{n}=\underset{y \in X}{\arg \min }\left[f(y)+\frac{1}{2 \lambda_{n}} d^{2}\left(y, x_{n}\right)\right], \\
y_{n}=\beta_{n} z_{n} \oplus\left(1-\beta_{n}\right) w_{n}, \quad w_{n} \in S y_{n}, \\
x_{n+1}=\alpha_{n} x_{n} \oplus\left(1-\alpha_{n}\right) T z_{n}, \quad \forall n \in N,
\end{array}\right.
$$

where $\alpha_{n}, \beta_{n} \in[0,1], T$ is a single-valued nonexpansive mapping, and $S$ is a multi-valued nonexpansive mapping.
Motivated and inspired by the above research work, Ishikawa iteration process, multivalued mapping, nonexpansive mapping, and convex function are considered as some elements for a new idea; then, the plan is to make use of these elements to reconstruct an algorithm; thus in this article, a modified proximal point algorithm involving a convex function and two nonexpansive mappings will be proposed. Under some suitable conditions, the convergence of the proposed algorithm is studied and its convergence analysis in the end is given.

\section{Preliminaries}

If $z, x$, and $y$ are three points in CAT(0) spaces and if $((x \oplus y) / 2)$ is the midpoint of a geodesic segment $[x, y]$, then the CAT (0) inequality implies

$$
d^{2}\left(z, \frac{x \oplus y}{2}\right) \leq \frac{1}{2} d^{2}(z, x)+\frac{1}{2} d^{2}(z, y)-\frac{1}{4} d^{2}(x, y),
$$

which is the $(C N)$ inequality (see [19]).

Let $X$ be a CAT (0) space; a subset $D \in X$ is convex, if for any $x, y \in D,[x, y] \in D$, where $[x, y]=\{\lambda x \oplus(1-\lambda) y: 0 \leq$ $\lambda \leq 1\}$ and $[x, y]$ is the unique geodesic joining $x$ and $y$. Indeed, a geodesic space $(X, d)$ is said to be a CAT $(0)$ space, if and only if the inequality $\left(\left(C N^{*}\right)\right.$ inequality [20]),

$$
\begin{aligned}
d^{2}((1-\lambda) x \oplus \lambda y, z) \leq & (1-\lambda) d^{2}(x, z) \\
& +\lambda d^{2}(y, z)-\lambda(1-\lambda) d^{2}(x, y)
\end{aligned}
$$

is satisfied for all $x, y, z \in X$ and $\lambda \in[0,1]$. Moreover, if $x, y$, and $z$ are points in a $\operatorname{CAT}(0)$ space $(X, d)$ and $\lambda \in[0,1]$, then

$$
d((1-\lambda) x \oplus \lambda y, z) \leq(1-\lambda) d(x, z)+\lambda d(y, z) .
$$

Let $\mathrm{CB}(D)$ and $\mathrm{KC}(D)$ denote the families of nonempty closed bounded subsets and compact convex subsets of $D$, respectively. The Pompeiu-Hausdorff distance [21] on $\mathrm{CB}(D)$ is defined by

$$
H(A, B)=\max \left\{\sup _{x \in A} \operatorname{dist}(x, B), \sup _{y \in B} \operatorname{dist}(y, A)\right\},
$$

for $A, B \in \mathrm{CB}(D)$, where $\operatorname{dist}(x, D)=\inf \{d(x, y): y \in D\}$ is the distance from a point $x$ to a subset $D$. Let $S: D \longrightarrow$ $\mathrm{CB}(D)$ be a multivalued mapping. An element $x \in D$ is said to be a fixed point of mapping $S$, if an element $x \in S x$. The set of fixed points of mapping $S$ is denoted by $F(S)$; in brief, $F(S)=\{x \in D: x \in S x\}$. More references for a multivalued mapping can be seen in $[18,22,23]$.

Definition 1 (see [18]). A single-valued mapping $T: D \longrightarrow$ $D$ is nonexpansive if $\forall x, y \in D, d(T x, T y) \leq d(x, y)$.

Definition 2 (see [18]). A multivalued mapping $S: D \longrightarrow$ $\mathrm{CB}(D)$ is nonexpansive if $\forall x, y \in D, H(S x, S y) \leq d(x, y)$.

Definition 3 (see [18]). Let $\left\{x_{n}\right\}$ be a bounded sequence in a CAT (0) space $X$. For any $x \in X$, we define a mapping 
$r\left(\cdot,\left\{x_{n}\right\}\right): X \longrightarrow[0, \infty)$ by $r\left(x,\left\{x_{n}\right\}\right)=\lim _{n \longrightarrow \infty} \sup d(x$, $\left.x_{n}\right)$ : (i) the asymptotic radius of $\left\{x_{n}\right\}$ is given by $r\left(\left\{x_{n}\right\}\right)$ $=\inf \left\{r\left(x,\left\{x_{n}\right\}\right): x \in X\right\}$; (ii) the asymptotic center of $\left\{x_{n}\right\}$ is the set $A\left(\left\{x_{n}\right\}\right)=\left\{x \in X: r\left(x,\left\{x_{n}\right\}\right)=r\left(\left\{x_{n}\right\}\right)\right\}$.

The asymptotic center $A\left(\left\{x_{n}\right\}\right)$ in a complete CAT(0) space consists of exactly one point [24].

Definition 4 (see [18]). A sequence $\left\{x_{n}\right\}$ in a $\operatorname{CAT}(0)$ space $X$ is said to $\Delta$-converge to $x \in X$ if $x$ is the unique asymptotic center of every subsequence of $\left\{x_{n}\right\}$. In this case, we write $\Delta-\lim _{n \longrightarrow \infty} x_{n}=x$ and call $x$ as the $\Delta$-limit of $\left\{x_{n}\right\}$.

In fact, given $\left\{x_{n}\right\}$ in $X$ such that $\left\{x_{n}\right\} \Delta$-converges to $x$ and given $y \in X$ with $x \neq y, \quad \lim _{n \rightarrow \infty} \inf d\left(x_{n}, x\right)<$ $\lim _{n \longrightarrow \infty} \inf d\left(x_{n}, y\right)$. So, the Opial condition in Banach space is also satisfied in every CAT(0) space $X$.

Lemma 1 (see [25]). Every bounded sequence in a CAT(0) space has a $\Delta$-convergent subsequence.

Lemma 2 (see [26]). Let $D$ be a nonempty closed convex subset of a CAT (0) space X. If $\left\{x_{n}\right\}$ is a bounded sequence in $D$, then the asymptotic center of $\left\{x_{n}\right\}$ is in D.

Lemma 3 (see [20]). If $\left\{x_{n}\right\}$ is a bounded sequence in a complete CAT(0) space with $A\left(\left\{x_{n}\right\}\right)=\{x\},\left\{u_{n}\right\}$ is a subsequence of $\left\{x_{n}\right\}$ with $A\left(\left\{u_{n}\right\}\right)=\{u\}$, and the sequence $\left\{d\left(x_{n}, u\right)\right\}$ converges, then $x=u$.

Lemma 4 (see [20]). Let $D$ be a nonempty closed convex subset of a complete CAT (0) space $X$ and $T: D \longrightarrow D$ be a nonexpansive mapping. If $\left\{x_{n}\right\}$ is a bounded sequence in $D$ such that $\lim _{n \longrightarrow \infty} d\left(x_{n}, T x_{n}\right)=0$ and $\Delta-\lim _{n \longrightarrow \infty} x_{n}=x$, then $x=T x$.

Lemma 5 (see [12]). Let $(X, d)$ be a complete CAT (0) space and $f: X \longrightarrow(-\infty, \infty]$ be a proper convex and lower semicontinuous function. Then, the following identity holds:

$$
J_{\lambda} x=J_{\mu}\left(\frac{\lambda-\mu}{\lambda} J_{\lambda} x \oplus \frac{\mu}{\lambda} x\right), \quad \forall x \in X, \lambda>\mu>0,
$$

where $J_{\lambda}$ is the Moreau - Yosida resolvent of $f$.

Lemma 6 (see [27]). Let $(X, d)$ be a complete CAT (0) space and $f: X \longrightarrow(-\infty, \infty]$ be a proper convex and lower semicontinuous function. Then, for all $x, y \in X$ and $\lambda>0$, the following inequality holds:

$\frac{1}{2 \lambda} d^{2}\left(J_{\lambda} x, y\right)-\frac{1}{2 \lambda} d^{2}(x, y)+\frac{1}{2 \lambda} d^{2}\left(x, J_{\lambda} x\right)+f\left(J_{\lambda} x\right) \leq f(y)$,

where $J_{\lambda}$ is the Moreau-Yosida resolvent of $f$.

Lemma 7 (see $[28,29])$. Let $X$ be a CAT (0) space and D be a nonempty closed and convex subset of $X$. Let $\left\{x_{i}\right\}_{i=1}^{n}$ be any finite subset of $D$ and $\alpha_{i} \in(0,1), i=1,2, \ldots, n$ such that $\sum_{i=1}^{n} \alpha_{i}=1$. Then, the following inequalities hold: (i) $d\left(\oplus_{i=1}^{n} \alpha_{i} x_{i}, z\right) \leq \sum_{i=1}^{n} \alpha_{i} d\left(x_{i}, z\right), \forall z \in D$

(ii) $d^{2}\left(\oplus_{i=1}^{n} \alpha_{i} x_{i}, z\right) \leq \sum_{i=1}^{n} \alpha_{i} d^{2}\left(x_{i}, z\right)-\sum_{i, j=1, i \neq j}^{n} d^{2}\left(x_{i}, x_{j}\right)$, $\forall z \in D$

\section{Main Results}

Theorem 1. Let $(X, d)$ denote a $C A T(0)$ space and be complete, assuming that the subset $D \subseteq X$ is nonempty, closed, and convex. Suppose that $S: D \longrightarrow C B(D)$ is a multivalued nonexpansive mapping, $T$ is a single-valued nonexpansive mapping, and $f: D \longrightarrow(-\infty, \infty]$ is a proper convex and lower semicontinuous function. Suppose that the set

$$
\Omega=F(T) \cap F(S) \cap \underset{y \in C}{\arg \min } f(y) \neq \varnothing
$$

and $S p=\{p\}$ for $p \in \Omega$. For $x_{1} \in D$, let the sequence $\left\{x_{n}\right\}$ be defined by

$$
\left\{\begin{array}{l}
w_{n}=\underset{y \in C}{\arg \min }\left[f(y)+\frac{1}{2 \lambda_{n}} d^{2}\left(y, x_{n}\right)\right], \\
x_{n+1}=\alpha_{n} x_{n} \oplus \beta_{n} p_{n} \oplus \gamma_{n} T p_{n}, \quad p_{n} \in S w_{n},
\end{array}\right.
$$

the sequences $\left\{\alpha_{n}\right\},\left\{\beta_{n}\right\},\left\{\gamma_{n}\right\} \subseteq(0,1)$, and $0<a \leq \alpha_{n}, \beta_{n}, \gamma_{n} \leq$ $b<1, \alpha_{n}+\beta_{n}+\gamma_{n}=1 n \in N$, and $\left\{\lambda_{n}\right\}$ is a sequence such that $\lambda_{n} \geq \lambda>0$ for all $n \in N$ and some $\lambda$. Then, the following statements hold:

(i) $\lim _{n \rightarrow \infty} d\left(x_{n}, p\right)$ exists for all $p \in \Omega$

(ii) $\lim _{n \rightarrow \infty} d\left(x_{n}, w_{n}\right)=0$

(iii) $\lim _{n \rightarrow \infty} d\left(x_{n}, T x_{n}\right)=0$

(iv) $\lim _{n \rightarrow \infty} \operatorname{dist}\left(x_{n}, S x_{n}\right)=0$

(v) $\lim _{n \rightarrow \infty} d\left(x_{n}, J_{\lambda} x_{n}\right)=0$

Proof. Let $p \in \Omega$; then, we get that $p=T p \in S p$ and $f(p) \leq f(y)$ for all $y \in D$. Thus, it shows that

$$
f(p)+\frac{1}{2 \lambda_{n}} d^{2}(p, p) \leq f(y)+\frac{1}{2 \lambda_{n}} d^{2}(y, p), \quad \forall y \in D,
$$

and hence, $p=J_{\lambda} p$ for each $n \in N$.

(i) First of all, the first step is to prove the fact that for all $p \in \Omega, \lim _{n \longrightarrow \infty} d\left(x_{n}, p\right)$ exists. Since $w_{n}=J_{\lambda_{n}} x_{n}$, with the nonexpansiveness of $J_{\lambda_{n}}$, then

$$
d\left(w_{n}, p\right)=d\left(J_{\lambda_{n}} x_{n}, J_{\lambda_{n}} p\right) \leq d\left(x_{n}, p\right) .
$$

For $p \in \Omega$, by $p \in S p$, (17), and Lemma 7, we have

$$
\begin{aligned}
d\left(x_{n+1}, p\right) & =d\left(\alpha_{n} x_{n} \oplus \beta_{n} p_{n} \oplus \gamma_{n} T p_{n}, p\right) \\
& \leq \alpha_{n} d\left(x_{n}, p\right)+\beta_{n} d\left(p_{n}, p\right)+\gamma_{n} d\left(T p_{n}, p\right) \\
& \leq \alpha_{n} d\left(x_{n}, p\right)+\beta_{n} H\left(S w_{n}, S p\right)+\gamma_{n} d\left(T p_{n}, p\right) \\
& \leq \alpha_{n} d\left(x_{n}, p\right)+\beta_{n} d\left(w_{n}, p\right)+\gamma_{n} d\left(p_{n}, p\right) \\
& \leq \alpha_{n} d\left(x_{n}, p\right)+\beta_{n} d\left(w_{n}, p\right)+\gamma_{n} d\left(w_{n}, p\right) \\
& \leq \alpha_{n} d\left(x_{n}, p\right)+\left(1-\alpha_{n}\right) d\left(w_{n}, p\right) \\
& \leq d\left(x_{n}, p\right) .
\end{aligned}
$$


This shows that the sequence $\left\{d\left(x_{n}, p\right)\right\}$ is decreasing and bounded. So, the limit $\lim _{n \rightarrow \infty} d$ $\left(x_{n}, p\right)$ exists for all $p \in \Omega$.

(ii) Next, prove that $\lim _{n \longrightarrow \infty} d\left(x_{n}, w_{n}\right)=0$. Now, we let

$$
\lim _{n \rightarrow \infty} d\left(x_{n}, p\right)=c
$$

where $c$ is a constant in $[0, \infty)$ and $p \in \Omega$. In fact, by the inequality of Lemma 6 , it implies that

$$
\begin{aligned}
& \frac{1}{2 \lambda_{n}} d^{2}\left(w_{n}, p\right)-\frac{1}{2 \lambda_{n}} d^{2}\left(x_{n}, p\right)+\frac{1}{2 \lambda_{n}} d^{2}\left(w_{n}, x_{n}\right) \\
& \quad \leq f(p)-f\left(w_{n}\right) .
\end{aligned}
$$

Because of $f(p) \leq f\left(w_{n}\right)$ for all $n \geq 1$, it follows that

$$
d^{2}\left(w_{n}, x_{n}\right) \leq d^{2}\left(x_{n}, p\right)-d^{2}\left(w_{n}, p\right)
$$

Since $\lim _{n \rightarrow \infty} d\left(x_{n}, p\right)=c$, in order to show the fact that $\lim _{n \longrightarrow \infty} d\left(x_{n}, w_{n}\right)=0$, it is sufficient to show that

$$
\lim _{n \rightarrow \infty} d\left(w_{n}, p\right)=c
$$

From (18), we have

$$
d\left(w_{n}, p\right) \geq \frac{1}{1-\alpha_{n}}\left[d\left(x_{n+1}, p\right)-\alpha_{n} d\left(x_{n}, p\right)\right]
$$

Then, this shows that

$$
\begin{aligned}
& \lim _{n \longrightarrow \infty} \inf d\left(w_{n}, p\right) \\
& \geq \lim _{n \longrightarrow \infty} \inf \left\{\frac{1}{1-\alpha_{n}}\left[d\left(x_{n+1}, p\right)-\alpha_{n} d\left(x_{n}, p\right)\right]\right\}=c .
\end{aligned}
$$

At the same time, from (17), we get that

$\lim _{n \longrightarrow \infty} \operatorname{supd}\left(w_{n}, p\right) \leq \lim _{n \longrightarrow \infty} \operatorname{supd}\left(x_{n}, p\right)=c$.

Thus, from (24) and (25), it is implied that

$$
\lim _{n \longrightarrow \infty} d\left(w_{n}, p\right)=c \text {. }
$$

Then, from (19), (21), and (26), it can be shown that

$$
\lim _{n \rightarrow \infty} d\left(x_{n}, w_{n}\right)=0 \text {. }
$$

(iii) Next, prove that $\lim _{n \longrightarrow \infty} d\left(x_{n}, T x_{n}\right)=0$. By Lemma 7, we have

$$
\begin{aligned}
d^{2}\left(x_{n+1}, p\right)= & d^{2}\left(\alpha_{n} x_{n} \oplus \beta_{n} p_{n} \oplus \gamma_{n} T p_{n}, p\right) \\
\leq & \alpha_{n} d^{2}\left(x_{n}, p\right)+\beta_{n} d^{2}\left(p_{n}, p\right)+\gamma_{n} d^{2}\left(T p_{n}, p\right) \\
& \quad-\alpha_{n} \beta_{n} d^{2}\left(x_{n}, p_{n}\right)-\alpha_{n} \gamma_{n} d^{2}\left(x_{n}, T p_{n}\right)-\beta_{n} \gamma_{n} d^{2}\left(p_{n}, T p_{n}\right) \\
\leq & \alpha_{n} d^{2}\left(x_{n}, p\right)+\beta_{n} H^{2}\left(S w_{n}, S p\right)+\gamma_{n} d^{2}\left(T p_{n}, T p\right) \\
& \quad-\alpha_{n} \beta_{n} d^{2}\left(x_{n}, p\right)-\alpha_{n} \gamma_{n} d\left(x_{n}, T p_{n}\right)-\beta_{n} \gamma_{n} d^{2}\left(p_{n}, T p_{n}\right) \\
\leq & \alpha_{n} d^{2}\left(x_{n}, p\right)+\beta_{n} d^{2}\left(w_{n}, p\right)+\gamma_{n} d^{2}\left(p_{n}, p\right) \\
& \quad-\alpha_{n} \beta_{n} d^{2}\left(x_{n}, p\right)-\alpha_{n} \gamma_{n} d\left(x_{n}, T p_{n}\right)-\beta_{n} \gamma_{n} d^{2}\left(p_{n}, T p_{n}\right) \\
\leq & d^{2}\left(x_{n}, p\right)-\alpha_{n} \beta_{n} d^{2}\left(x_{n}, p_{n}\right)-\alpha_{n} \gamma_{n} d\left(x_{n}, T p_{n}\right)-\beta_{n} \gamma_{n} d^{2}\left(p_{n}, T p_{n}\right),
\end{aligned}
$$

that is,

$$
\begin{aligned}
& \alpha_{n} \beta_{n} d^{2}\left(x_{n}, p_{n}\right)+\alpha_{n} \gamma_{n} d\left(x_{n}, T p_{n}\right) \\
& \quad+\beta_{n} \gamma_{n} d^{2}\left(p_{n}, T p_{n}\right) \leq d^{2}\left(x_{n}, p\right)-d^{2}\left(x_{n+1}, p\right) .
\end{aligned}
$$$$
d\left(x_{n}, T x_{n}\right) \leq d\left(x_{n}, T p_{n}\right)+d\left(T p_{n}, T x_{n}\right)
$$$$
\leq d\left(x_{n}, T p_{n}\right)+d\left(p_{n}, x_{n}\right) \text {. }
$$

Then from (19) and (29), we get

$$
\begin{aligned}
& \lim _{n \longrightarrow \infty} d\left(x_{n}, p_{n}\right)=0, \\
& \lim _{n \longrightarrow \infty} d\left(x_{n}, T p_{n}\right)=0, \\
& \lim _{n \longrightarrow \infty} d\left(p_{n}, T p_{n}\right)=0 .
\end{aligned}
$$

From (30) and (31), it can be shown that

$$
\lim _{n \longrightarrow \infty} d\left(x_{n}, T x_{n}\right)=0 \text {. }
$$

(iv) Thus,

$$
\begin{aligned}
\operatorname{dist}\left(x_{n}, S x_{n}\right) & \leq d\left(x_{n}, p_{n}\right)+\operatorname{dist}\left(p_{n}, S x_{n}\right) \\
& \leq d\left(x_{n}, p_{n}\right)+H\left(S w_{n}, S x_{n}\right) \\
& \leq d\left(x_{n}, p_{n}\right)+d\left(w_{n}, x_{n}\right) .
\end{aligned}
$$

Therefore, from (30), we can obtain

From (27), (30), and (33), it is implied that 


$$
\operatorname{dist}\left(x_{n}, S x_{n}\right)=0 .
$$

(v) Since $\lambda_{n} \geq \lambda>0$, making use of $w_{n}=J_{\lambda_{n}} x_{n}$, the nonexpansiveness of $J_{\lambda}$, and Lemma 5 , it can be seen that

$$
\begin{aligned}
d\left(x_{n}, J_{\lambda} x_{n}\right) \leq & d\left(x_{n}, w_{n}\right)+d\left(w_{n}, J_{\lambda} x_{n}\right) \\
& \leq d\left(x_{n}, w_{n}\right)+d\left(J_{\lambda_{n}} x_{n}, J_{\lambda} x_{n}\right) \\
& =d\left(x_{n}, w_{n}\right)+d\left(J_{\lambda}\left(\frac{\lambda_{n}-\lambda}{\lambda_{n}} J_{\lambda_{n}} x_{n} \oplus \frac{\lambda}{\lambda_{n}} x_{n}\right), J_{\lambda} x_{n}\right) \\
& \leq d\left(x_{n}, w_{n}\right)+\frac{\lambda_{n}-\lambda}{\lambda_{n}} d\left(J_{\lambda_{n}} x_{n}, x_{n}\right)+\frac{\lambda}{\lambda_{n}} d\left(x_{n}, x_{n}\right) \\
& =\left(2-\frac{\lambda}{\lambda_{n}}\right) d\left(x_{n}, w_{n}\right) .
\end{aligned}
$$

So, this implies the fact that the limit

$$
\lim _{n \rightarrow \infty} d\left(x_{n}, J_{\lambda} x_{n}\right)=0
$$

This completes the proof.

Theorem 2. Let $D$ be a nonempty closed convex subset of a complete CAT(0) space $(X, d)$. Let function $f: D \longrightarrow(-\infty$, $\infty$ ] be a proper convex and lower semicontinuous function, $T$ be a nonexpansive single-valued mapping, and $S: D \longrightarrow K C$

(D) be a multivalued nonexpansive mapping. Suppose that

$$
\Omega=F(T) \cap F(S) \cap \underset{y \in D}{\arg \min } f(y) \neq \varnothing
$$

and $S p=\{p\}$ for $p \in \Omega$. For $x_{1} \in D$, let the sequence $\left\{x_{n}\right\}$ be defined by (15), the sequences $\left\{\alpha_{n}\right\},\left\{\beta_{n}\right\},\left\{\gamma_{n}\right\} \in(0,1)$, and $0<a \leq \alpha_{n}, \beta_{n}, \gamma_{n} \leq b<1, \alpha_{n}+\beta_{n}+\gamma_{n}=1 n \in N$. In addition, $\left\{\lambda_{n}\right\}$ is a sequence such that $\lambda_{n} \geq \lambda>0$ for all $n \in N$ and some $\lambda$. Then, the sequence $\left\{x_{n}\right\} \Delta$-converges to a point in $\Omega$.

Proof. Let $\omega_{\Delta}=\bigcup A\left(\left\{u_{n}\right\}\right)$, where the union is taken over all subsequences $\left\{u_{n}\right\}$ of $\left\{x_{n}\right\}$. Let $p \in \omega_{\Delta}\left(x_{n}\right)$. Then, there exists a subsequence $\left\{u_{n}\right\}$ of $\left\{x_{n}\right\}$ such that $A\left(\left\{u_{n}\right\}\right)=\{p\}$. $D \in X$ is a nonempty closed convex subset, and it is easy to know from Theorem 1 that the sequence $\left\{x_{n}\right\}$ is bounded. Then, by Lemmas 1 and 2, it can be shown that there exists a subsequence $\left\{v_{n}\right\}$ of $\left\{u_{n}\right\}$ such that

$$
\Delta-\lim _{n \longrightarrow \infty} v_{n}=v \in D \text {. }
$$

From Theorem 1 (iii) and (v), we have $\lim _{n \rightarrow \infty} d\left(v_{n}\right.$, $\left.T v_{n}\right)=0$ and $\lim _{n \longrightarrow \infty} d\left(v_{n}, J_{\lambda} v_{n}\right)=0$. Then, by the nonexpansiveness of $T$ and $J_{\lambda}$, through Lemma 4 , it can be shown that the fact that $v=T v=J_{\lambda} v$. Thus, we get

$$
v \in F(T) \cap F\left(J_{\lambda}\right)=F(T) \cap \underset{u \in D}{\arg \min } f(u) .
$$

Since the mapping $S$ is compact valued, for each $n \in N$, then there exist $r_{n} \in S v_{n}$ and $\gamma_{n} \in S v$ such that $d\left(v_{n}, r_{n}\right)=$ $\operatorname{dist}\left(v_{n}, S v_{n}\right)$ and $d\left(r_{n}, \gamma_{n}\right)=\operatorname{dist}\left(r_{n}, S v\right)$. By Theorem 1 (iv), this implies the fact that

$$
\lim _{n \rightarrow \infty} d\left(v_{n}, r_{n}\right)=0
$$

Owing to the fact $S v$ is compact, there exists a subsequence $\left\{\gamma_{n_{i}}\right\}$ of $\left\{\gamma_{n}\right\}$ such that $\lim _{i \longrightarrow \infty} \gamma_{n_{i}}=\gamma \in S v$. Thus, this shows that

$$
\begin{aligned}
\lim _{i \longrightarrow \infty} \sup d\left(v_{n_{i}}, \gamma\right) & \leq \lim _{i \longrightarrow \infty} \sup \left(d\left(v_{n_{i}}, r_{n_{i}}\right)+d\left(r_{n_{i}}, \gamma_{n_{i}}\right)+d\left(\gamma_{n_{i}}, \gamma\right)\right) \\
& \leq \lim _{i \longrightarrow \infty} \sup \left(d\left(v_{n_{i}}, r_{n_{i}}\right)+\operatorname{dist}\left(r_{n_{i}}, S v\right)+d\left(\gamma_{n_{i}}, \gamma\right)\right) \\
& \leq \lim _{i \longrightarrow \infty} \sup \left(d\left(v_{n_{i}}, r_{n_{i}}\right)+H\left(S r_{n_{i}}, S v\right)+d\left(\gamma_{n_{i}}, \gamma\right)\right) \\
& \leq \lim _{i \longrightarrow \infty} \sup \left(d\left(v_{n_{i}}, r_{n_{i}}\right)+d\left(r_{n_{i}}, v\right)+d\left(\gamma_{n_{i}}, \gamma\right)\right) \\
& =\lim _{i \longrightarrow \infty} \sup d\left(v_{n_{i}}, v\right) .
\end{aligned}
$$

Through (38) and the uniqueness of asymptotic centers, we can obtain that $v=\gamma \in S v$. Therefore, by (39), we can show that

$$
v \in F(T) \cap F(S) \cap \underset{u \in D}{\arg \min } f(u)=\Omega .
$$

It follows by Lemma 3 and Theorem 1 (i) that $p=v$, and hence, $\omega_{\Delta}\left(x_{n}\right) \subseteq \Omega$.

In order to show that $\left\{x_{n}\right\} \Delta$-converges to a point in $\Omega$, it suffices to show that $\omega_{\Delta}\left(x_{n}\right)$ consists of exactly one point. Suppose that $\left\{u_{n}\right\}$ is a subsequence of $\left\{x_{n}\right\}$ with $A\left(\left\{u_{n}\right\}\right)=$ $\left\{u^{*}\right\}$ and $A\left(\left\{x_{n}\right\}\right)=\{x\}$. Since $u^{*} \in \omega_{\Delta}\left(x_{n}\right) \subseteq \Omega$ and $\left\{d\left(x_{n}\right.\right.$, $\left.\left.u^{*}\right)\right\}$ converge, it implies by Lemma 3 that $x=u^{*}$.

This completes the proof.

Remark 1.

(i) The results of Shuntai and Phuaengrattana [18] and Cholamjiak [30] are extended and improved by Theorem 2. In fact, a new proximal point algorithm can be used for solving the constrained convex 
minimization problem as well as the fixed-point problem of a single-valued nonexpansive mapping and a multivalued nonexpansive mapping in a CAT(0) space.

(ii) Since every real Hilbert space $\mathrm{H}$ is a complete CAT(0) space, the above result can also be obtained in Hilbert spaces, so a convergence weakly theorem can be obtained in a real Hilbert space as follows.

Corollary 1. Let $D$ be a nonempty closed convex subset of a real Hilbert space $H$. Let $f: D \longrightarrow(-\infty, \infty]$ be a proper convex and lower semicontinuous function, $T$ be a singlevalued nonexpansive mapping, and $S: D \longrightarrow C B(D)$ be a multivalued nonexpansive mapping. Suppose that

$$
\Omega=F(T) \cap F(S) \cap \underset{y \in C}{\arg \min } f(y) \neq \varnothing
$$

and $S p=\{p\}, p \in \Omega$. For $x_{1} \in D$, let the sequence $\left\{x_{n}\right\}$ be defined by

$$
\left\{\begin{array}{l}
w_{n}=\underset{y \in C}{\arg \min }\left[f(y)+\frac{1}{2 \lambda_{n}} d^{2}\left(y, x_{n}\right)\right], \\
x_{n+1}=\alpha_{n} x_{n}+\beta_{n} p_{n}+\gamma_{n} T p_{n}, \quad p_{n} \in S w_{n},
\end{array}\right.
$$

the sequences $\left\{\alpha_{n}\right\},\left\{\beta_{n}\right\},\left\{\gamma_{n}\right\} \subseteq(0,1)$, and $0<a \leq \alpha_{n}, \beta_{n}, \gamma_{n} \leq$ $b<1, \alpha_{n}+\beta_{n}+\gamma_{n}=1 n \in N$, and $\left\{\lambda_{n}\right\}$ is a sequence such that $\lambda_{n} \geq \lambda>0, \forall n \in N$ and some $\lambda$. Then, the sequence $\left\{x_{n}\right\}$ converges weakly to an element in $\Omega$.

Remark 2. In fact, the construction of our proposed algorithm is rather peculiar and it is different from references [31-33]. Convex combination of the sequences $\left\{x_{n}\right\},\left\{p_{n}\right\}$, and $\left\{T p_{n}\right\}$ are given here, especially $p_{n} \in S w_{n}$, and $T p_{n}$ is the nonexpansive mapping $T$ that operates on the sequences $\left\{p_{n}\right\}$.

\section{Numerical Experiments}

In this section, a numerical example is given to illustrate reckoning the convergence of modified proximal point algorithm with iteration (15) by numerical experiment for supporting Theorems 1 and 2.

Let $X=\mathbb{R}^{2}$ with Euclidean norm and $D=\left\{x=\left(x^{(1)}\right.\right.$, $\left.\left.x^{(2)}\right) \in \mathbb{R}^{2}: 0 \leq x^{(1)}, x^{(2)} \leq 1\right\}$. For each $x=\left(x^{(1)}, x^{(2)}\right) \in D$, the concrete definition of nonexpansive mappings $T$ and $S$ is shown as

$$
\begin{aligned}
& T x=\left(\frac{2 x^{(1)}+1}{4}, \frac{x^{(2)}+7}{8}\right), \\
& S x=\left\{x^{(1)}\right\} \times\left[\frac{3 x^{(2)}+1}{4}, 1\right] .
\end{aligned}
$$

For each $x \in D$, assume that $\|x\|_{1}=\sum_{i=1}^{n}\left|x_{i}\right|$, and $\|x\|_{2}=$ $\sqrt{\sum_{i=1}^{n} x_{i}^{2}}$. The function $f: D \longrightarrow(-\infty, \infty)$ is defined in the following manner:

$$
f(x)=\|x\|_{1}+\frac{1}{2}\|x\|_{2}^{2}+(-1.5,-2) x+7 .
$$

From the fact that $T$ and $S$ are nonexpansive and $f$ is a proper convex lower semicontinuous function easy to prove, we skip their proofs here. Furthermore, by making use of the soft thresholding operator [34] and the proximity operator [35], let $\lambda_{n}=1$; we have

$$
\begin{aligned}
& J_{0} x=\underset{v \in D}{\arg \min }\left[f(v)+\frac{1}{2}\|v-x\|^{2}\right]=\operatorname{prox}_{f} x \\
& =\operatorname{prox}_{\left(\|\cdot\|_{1} / 2\right)}\left(\frac{x-(-1.5,-2)}{2}\right) \\
& =\left(\max \left\{\frac{\left|x^{(1)}+1.5\right|-1}{2}, 0\right\} \operatorname{sgn}\left(x^{(1)}+1.5\right), \max \left\{\frac{\left|x^{(2)}+2\right|-1}{2}, 0\right\} \operatorname{sgn}\left(x^{(2)}+2\right)\right),
\end{aligned}
$$

where $\operatorname{sgn}(\xi)$ is a signum function, that is,

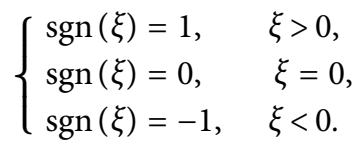

Further simplification of the proposed iterative algorithm is in the following expression:

$$
\left\{\begin{array}{l}
w_{n}=J_{0} x_{n}, \\
x_{n+1}=\alpha_{n} x_{n}+\beta_{n} p_{n}+\gamma_{n} T p_{n}, \quad p_{n} \in S w_{n},
\end{array}\right.
$$

where $x_{n}=\left(x_{n}^{(1)}, x_{n}^{(2)}\right)$ and $w_{n}=\left(w_{n}^{(1)}, w_{n}^{(2)}\right)$ are points in $\mathbb{R}^{2}$. In addition, we choose some points and the sequences of parameters as follows: 
TABLE 1: Numerical results of Algorithm (49).

\begin{tabular}{lccc}
\hline$n$ & $x_{n}=\left(x_{n}^{(1)}, x_{n}^{(2)}\right)$ & $\left\|x_{n}-x_{n-1}\right\|_{2}$ & $f\left(x_{n}\right)$ \\
\hline 1 & $(0.100000,0.200000)$ & 0.347205 & 6.525875 \\
3 & $(0.310620,0.705335)$ & 0.124269 & 6.400753 \\
5 & $(0.406401,0.884487)$ & 0.050838 & 6.379830 \\
7 & $(0.453275,0.954060)$ & 0.021717 & 6.375968 \\
9 & $(0.476569,0.981610)$ & 0.009587 & 6.375205 \\
11 & $(0.488219,0.992611)$ & 0.004359 & 6.375045 \\
13 & $(0.494066,0.997023)$ & 0.002034 & 6.375010 \\
15 & $(0.497008,0.998799)$ & 0.000970 & 6.375002 \\
17 & $(0.498490,0.999514)$ & 0.000471 & 6.375000 \\
19 & $(0.499237,0.999803)$ & 0.000231 & 6.375000 \\
21 & $(0.499614,0.999920)$ & 0.000115 & 6.375000 \\
23 & $(0.499805,0.999967)$ & 0.000057 & 6.375000 \\
25 & $(0.499901,0.999986)$ & 0.000028 & 6.375000 \\
27 & $(0.499950,0.999994)$ & 0.000014 & 6.375000 \\
29 & $(0.499974,0.999997)$ & 0.000007 & 6.375000 \\
31 & $(0.499987,0.999999)$ & 0.000003 & 6.375000 \\
33 & $(0.499993,0.999999)$ & 0.000001 & 6.375000 \\
35 & $(0.499996,0.999999)$ & 0.000000 & 6.375000 \\
\hline
\end{tabular}

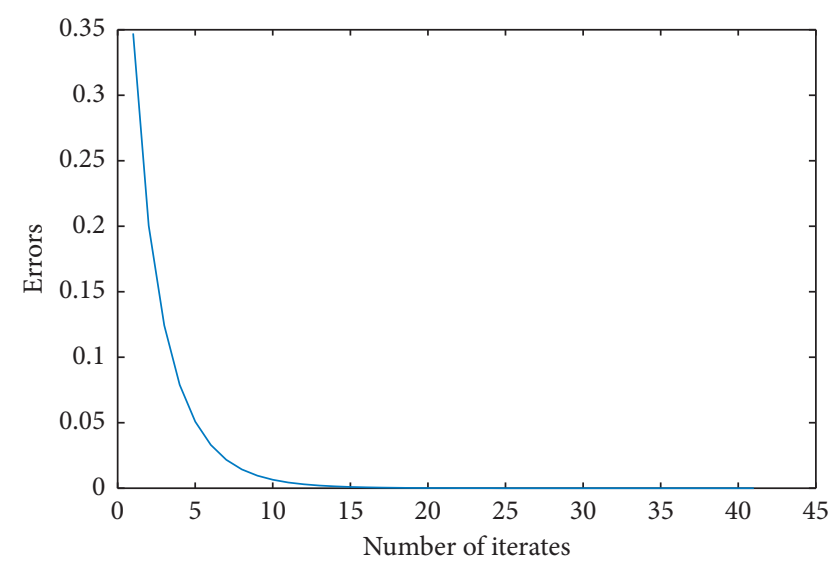

Figure 1: Number of iterations and errors.

$$
\begin{aligned}
& p_{n}=\left(w_{n}^{(1)}, \frac{3 w_{n}^{(2)}+1}{4}\right) \in S w_{n}, \\
& \alpha_{n}=\frac{50 n-3}{100 n}, \\
& \beta_{n}=\frac{35 n-4}{100 n}, \\
& \gamma_{n}=\frac{15 n+7}{100 n} .
\end{aligned}
$$

Next, we use Algorithm (49) with an initial point $x_{1}=$ $(0.1,0.2)$ and obtain numerical results in Table 1.

\section{Remark 3}

(i) From Figure 1, with the increase in the number of iterations, the errors between $x_{n}$ and $x_{n+1}$ decrease. When the iteration is 17 times, the minimum of $f$ is obtained. (ii) From Table 1 and Figure 1, it is observed that the sequence $\left\{x_{n}\right\}$ converges to a point $(0.5,1)$.

(iii) The point $(0.5,1)$ is a solution of the constrained convex minimization problems (46) and also a solution of the fixed-point problems of a pair of a nonexpansive single-valued mapping $T$ and a nonexpansive multivalued mapping $S$.

\section{Data Availability}

Some data obtained by the authors themselves were used to support this study.

\section{Conflicts of Interest}

All authors declare no conflicts of interest.

\section{Authors' Contributions}

All authors contributed equally to this study.

\section{Acknowledgments}

This work was jointly supported by the High-Level Talent Sailing Project of Yibin University (2021QH07).

\section{References}

[1] G. C. Bento, O. P. Ferreira, and P. R. Oliveira, "Proximal point method for a special class of nonconvex functions on Hadamard manifolds," Optimization, vol. 64, no. 2, pp. 289-319, 2015.

[2] B. Ahmadi Kakavandi and M. Amini, "Duality and subdifferential for convex functions on complete metric spaces," Nonlinear Analysis: Theory, Methods \& Applications, vol. 73, no. 10 , pp. $3450-3455,2010$.

[3] K. Sokhuma, " $\Delta$-convergence theorems for a pair of singlevalued and multivalued nonexpansive mappings in CAT(0) spaces," Journal of Mathematical Analysis and Applications, vol. 4, no. 2, pp. 23-31, 2013.

[4] U. F. Mayer, "Gradient flows on nonpositively curved metric spaces and harmonic maps," Communications in Analysis and Geometry, vol. 6, no. 2, pp. 199-253, 1998.

[5] S. H. Khan and M. Abbas, "Strong and $\triangle$-convergence of some iterative schemes in CAT(0) spaces," Computers \& Mathematics with Applications, vol. 61, no. 1, pp. 109-116, 2011.

[6] O. P. Ferreira and P. R. Oliveira, "Proximal point algorithm on Riemannian manifolds," Optimization, vol. 51, no. 2, pp. 257-270, 2002.

[7] M. Bridson and A. Haefliger, Metric Spaces of Non-positive Curvature, Springer, Berlin, Germany, 1999.

[8] G. C. Bento, O. P. Ferreira, and P. R. Oliveira, "Local convergence of the proximal point method for a special class of nonconvex functions on Hadamard manifolds," Nonlinear Analysis: Theory, Methods \& Applications, vol. 73, no. 2, pp. 564-572, 2010.

[9] O. Güler, "On the convergence of the proximal point algorithm for convex minimization," SIAM Journal on Control and Optimization, vol. 29, no. 2, pp. 403-419, 1991.

[10] D. Ariza-Ruiz, L. Leuştean, and G. López-Acedo, "Firmly nonexpansive mappings in classes of geodesic spaces," 
Transactions of the American Mathematical Society, vol. 366, no. 8, pp. 4299-4322, 2014.

[11] R. P. Agarwal, D. ORegan, and D. R. Sahu, "Iterative construction of fixed points of nearly asymptotically nonexpansive mappings," Journal of Nonlinear and Convex Analysis, vol. 8, pp. 61-79, 2007.

[12] J. Jost, "Convex functionals and generalized harmonic maps into spaces of non positive curvature," Commentarii Mathematici Helvetici, vol. 70, no. 1, pp. 659-673, 1995.

[13] B. Martinet, "Brève communication. Régularisation d'inéquations variationnelles par approximations successives," Revue française d'informatique et de recherche opérationnelle. Série rouge, vol. 4, no. R3, pp. 154-158, 1970.

[14] R. T. Rockafellar, "Monotone operators and the proximal point algorithm," SIAM Journal on Control and Optimization, vol. 14 , no. 5, pp. 877-898, 1976.

[15] M. Bacak, "The proximal point algorithm in metric spaces," Israel Journal of Mathematics, vol. 194, pp. 689-701, 2013.

[16] W. R. Mann, "Mean value methods in iteration," Proceedings of the American Mathematical Society, vol. 4, no. 3, p. 506, 1953.

[17] S. Ishikawa, "Fixed points by a new iteration method," Proceedings of the American Mathematical Society, vol. 44, no. 1, p. $147,1974$.

[18] S. Suantai and W. Phuengrattana, "Proximal point Algorithms for a hybrid pair of nonexpansive single-valued and multi-valued mappings in geodesic metric spaces," Mediterranean Journal of Mathematics, vol. 14, no. 2, p. 62, 2017.

[19] F. Bruhat and J. Tits, "Groupes réductifs sur un corps local," Publications Mathématiques de L'IHÉS, vol. 41, no. 1, pp. 5-251, 1972.

[20] S. Dhompongsa and B. Panyanak, "On $\triangle$-convergence theorems in CAT(0) spaces," Computers \& Mathematics with Applications, vol. 56, no. 10, pp. 2572-2579, 2008.

[21] R. T. Rockafellar and R. J.-B. Wets, Variational Analysis, Springer, Berlin, Germany, 2005.

[22] G. Durmaz and I. AltunOn, "Nonlinear set-valued $\Theta$-contractions," Bulletin of the Malaysian Mathematical Sciences Society, 2018.

[23] I. Altun and G. Minak, "On fixed point theorems for multivalued mappings of feng-liu type," Bulletin of the Korean Mathematical Society, vol. 52, no. 6, pp. 1901-1910, 2015.

[24] S. Dhompongsa, W. A. Kirk, and B. Sims, "Fixed points of uniformly Lipschitzian mappings," Nonlinear Analysis: Theory, Methods \& Applications, vol. 65, no. 4, pp. 762-772, 2006.

[25] W. A. Kirk and B. Panyanak, "A concept of convergence in geodesic spaces," Nonlinear Analysis: Theory, Methods \& Applications, vol. 68, no. 12, pp. 3689-3696, 2008.

[26] S. Dhompongsa, W. A. Kirk, and B. Panyanak, "Nonexpansive set-valued mappings in metric and Banach spaces," J. Nonlinear Convex Anal.vol. 8, pp. 35-45, 2007.

[27] L. Ambrosio, N. Gigli, and G. Savare, Gradient Flows in Metric Spaces and in the Space of Probability Measures, Lectures in Mathematics ETH Zrich, Birkhuser, Basel, Switzerland, 2nd edition, 2008.

[28] J. F. Tang, S. S. Chang, and J. Dong, "Strong convergence theorems of Cesaro-type means for nonexpansive mapping in CAT(0) spaces," Fixed Point Theory and Applications, vol. 100, 2015.

[29] C. E. Chidume, A. U. Bello, and P. Ndambomve, "Strong and $F$-convergence theorems for common fixed points of a finite family of multivalued demicontractive mappings in CAT(0) spaces," Abstract and Applied Analysis, 2014.
[30] P. Cholamjiak, "The modified proximal point algorithm in CAT(0) spaces," Optimization Letters, vol. 9, no. 7, pp. 1401-1410, 2015.

[31] Y. Shehu, "Weak and linear convergence of proximal point algorithm with reflections," Journal of Nonlinear and Convex Analysis, vol. 22, pp. 299-307, 2021.

[32] S. Y. Cho, "A convergence theorem for generalized mixed equilibrium problems and multivalued asymptotically nonexpansive mappings," Journal of Nonlinear and Convex Analysis, vol. 21, pp. 1017-1026, 2020.

[33] J. Fan, X. Qin, and B. Tan, “Tseng's extragradient algorithm for pseudomonotone variational inequalities on Hadamard manifolds," Applicable Analysis, pp. 1-14, 2021.

[34] E. T. Hale, W. Yin, and Y. Zhang, "A fixed-point continuation method for 11-regularized minimization with applications to compressed sensing," Tech.rep.,CAAM, 2007.

[35] P. L. Combettes and J.-C. Pesquet, "Proximal splitting methods in signal processing," in Fixed-Point Algorithms for Inverse Problems in Science and Engineering, Springer Optimization and Its Applications, H. H. Bauschke, R. Burachik, P. L. Combettes, V. Elser, D. R. Luke, and H. Wolkowicz, Eds., Springer, New York, pp. 185-212, 2011. 